\title{
Development of a dry reagent-based triplex PCR for the detection of toxigenic and non-toxigenic Vibrio cholerae
}

Correspondence

Pattabhiraman Lalitha

lalithapattabhiraman@gmail.com

Received 22 October 2010

Accepted 22 December 2010

\author{
Ang Lim Chua, ${ }^{1}$ Husni Tan Elina, ${ }^{2}$ Boon Huat Lim, ${ }^{1}$ Chan Yean Yean, ${ }^{2}$ \\ Manickam Ravichandran ${ }^{3}$ and Pattabhiraman Lalitha ${ }^{3}$ \\ ${ }^{1}$ School of Health Sciences, Universiti Sains Malaysia, 16150 Kubang Kerian, Kelantan, Malaysia \\ ${ }^{2}$ School of Medical Sciences, Universiti Sains Malaysia, 16150 Kubang Kerian, Kelantan, Malaysia \\ ${ }^{3}$ Department of Biotechnology, Faculty of Applied Sciences, AIMST University, Semeling, \\ 08100 Bedong, Kedah, Malaysia
}

\begin{abstract}
Vibrio cholerae has caused severe outbreaks of cholera worldwide with thousands of recorded deaths annually. Molecular diagnosis for cholera has become increasingly important for rapid detection of cholera as the conventional methods are time-consuming and labour intensive. However, traditional PCR tests still require cold-chain transportation and storage as well as trained personnel to perform, which makes them user-unfriendly. The aim of this study was to develop a thermostabilized triplex PCR test for cholera which is in a ready-to-use form and requires no cold chain. The PCR test specifically detects both toxigenic and non-toxigenic strains of $V$. cholerae based on the cholera toxin $A(c t x A)$ and outer-membrane lipoprotein $(/ o / B)$ genes. The thermostabilized triplex PCR also incorporates an internal amplification control that helps to check for PCR inhibitors in samples. PCR reagents and the specific primers were lyophilized into a pellet form in the presence of trehalose, which acts as an enzyme stabilizer. The triplex PCR was validated with 174 bacteria-spiked stool specimens and was found to be $100 \%$ sensitive and specific. The stability of the thermostabilized PCR was evaluated using the $\mathrm{Q}_{10}$ method and it was found to be stable for approximately 7 months at $24{ }^{\circ} \mathrm{C}$. The limit of detection of the thermostabilized triplex PCR assay was $2 \times 10^{4}$ c.f.u. at the bacterial cell level and $100 \mathrm{pg}$ DNA at the genomic DNA level, comparable to conventional PCR methods. In conclusion, a rapid thermostabilized triplex PCR assay was developed for detecting toxigenic and non-toxigenic $V$. cholerae which requires minimal pipetting steps and is cold chain-free.
\end{abstract}

\section{INTRODUCTION}

Cholera is an acute intestinal infection characterized mainly by rapid onset of severe watery diarrhoea, vomiting and rapid dehydration, which can lead to death if left untreated. The disease is caused by the Vibrio cholerae bacterium, mainly of serogroups O1 and O139. This disease caused several pandemics throughout the 19th century and continues to cause severe outbreaks around the world, especially in Asia and Africa (Wachsmuth et al., 1994). In 2008 alone, the World Health Organization recorded a total of 190130 reported cases including 5143 deaths with the African continent representing the bulk (WHO, 2009).

Due to the clinical and epidemiological severity of cholera, rapid diagnosis of $V$. cholerae in clinical samples, water and food is very critical so that appropriate monitoring and effective preventive measures can be undertaken to prevent a cholera outbreak. The conventional detection methods

Abbreviation: LoD, limit of detection. for diagnosis of cholera based on culture, microscopy and biochemical testing (CDC, 1999) are limited by the speed of detection and sensitivity and specificity levels.

Molecular methods, especially PCR, have become more important in the diagnosis of cholera and have greatly improved the speed, sensitivity and specificity of diagnostic tests, which in turn facilitates early and informed decisionmaking related to patient management, infection control, treatment and prevention (Yang \& Rothman, 2004). Early PCR tests were developed to target the cholera enterotoxin operon since cholera outbreaks are associated with enterotoxin-producing $V$. cholerae (Fields et al., 1992; Koch et al., 1993; Shirai et al., 1991). A multiplexed PCR was later developed to identify biotypes of $V$. cholerae $\mathrm{O} 1$ based on the haemolysin toxin gene ( hylA) gene and also the enterotoxin (Shangkuan et al., 1995). With the emergence of serogroup O139 of $V$. cholerae, PCR tests have also been developed to detect this serogroup (Albert et al., 1997; Lipp et al., 2003; Rivera et al., 1995). 
Other $V$. cholerae genes, such as the zonula occludens toxin $(z o t)$, accessory cholera enterotoxin (ace), toxin-coregulated pilus $(t c p)$, repeats in toxin $(r t x)$ and heat-stable enterotoxin (sto) virulence genes, have also been targeted for amplification in both monoplex and multiplex PCR tests (Fields et al., 1992; Koch et al., 1993; Rivera et al., 1995; Singh et al., 2001). However, these tests cannot detect environmental non-O1/ non-O139 serogroups of $V$. cholerae, which do not possess the toxigenic or other virulence genes but are still responsible for sporadic and localized outbreaks of cholera-like diarrhoea (Kaper et al., 1995). A PCR test designed based on an outer-membrane protein $(o m p W)$ gene was able to detect non-O1/non-O139 strains of $V$. cholerae (Nandi et al., 2000), but showed some cross-reaction with Vibrio mimicus. However, another PCR test developed to detect all serogroups, including non-O1/non-O139 serogroups, of $V$. cholerae based on the outer membrane lipoprotein (lolB) gene was highly specific and could discriminate other Vibrio species (Lalitha et al., 2008).

Conventional PCR tests have little use in resource-limited areas due to several factors such as the need for skilled personnel in the preparation of PCR, and the requirement of cold-chain transportation and storage. PCR tests usually involve handling micro volumes, where operator variability could cause a slight inaccuracy of just $1 \mu$, which can detrimentally affect a test result. Furthermore, PCR is compounded by the multiple pipetting steps of small volumes of reagents, which may lead to errors. These factors prevented the utilization of PCR as the diagnostic tool of choice for cholera, even though PCR provides higher sensitivity and specificity.

In this study, a thermostabilized triplex PCR test was developed for the detection of $V$. cholerae that is cold chain-free and requires only the simple addition of DNA samples and PCR-grade (DNase-free) water to perform the test. A triplex PCR with specific primers was designed for the $V$. cholerae lolB gene (Lalitha et al., 2008), the cholera toxin A gene $\operatorname{ctxA}$ and an internal amplification control (IC) for monitoring PCR inhibitors. The whole PCR mix with the specific primers was thermostabilized into a dried pellet, using a similar technique as described in previous studies (Aziah et al., 2007; Klatser et al., 1998). The readyto-use dried reagent mix was analysed for the limit of detection (LoD) and evaluated with spiked stool samples to mimic clinical specimens.

\section{METHODS}

Bacterial strains. Bacterial isolates used in this study were acquired from the Center for Southeast Asia Studies, Kyoto University, Japan; the Communicable Diseases Hospital, India; the National Institute of Cholera and Diseases (NICED), India; the National Public Health Laboratory, Ministry of Health, Malaysia; the Institute for Medical Research (IMR), Malaysia; and the Hospital Universiti Sains Malaysia, Malaysia. The bacterial isolates consisted of $102 \mathrm{~V}$. cholerae strains [O1 $(n=86), \mathrm{O} 139(n=12)$ and non-O1/non-O139 $(n=4)$ serogroups], 5 other Vibrio species [V. parahaemolyticus $(n=1), V$. fluvialis $(n=1), V$. furnissii $(n=1), V$. mimicus $(n=1)$ and $V$. vulnificus $(n=1)], 36$ other
Gram-negative bacteria [Acinetobacter baumannii $(n=1)$, Acinetobacter species $(n=1)$, Aeromonas hydrophila $(n=1)$, Burkholderia pseudomallei $(n=1)$, Citrobacter freundii $(n=1)$, Enterobacter cloacae $(n=1)$, Escherichia coli $(n=5)$, Klebsiella pneumoniae $(n=1)$, Klebsiella species $(n=2)$, Morganella morganii $(n=1)$, Proteus mirabilis $(n=1)$, Proteus vulgaris $(n=1)$, Providencia stuartii $(n=1)$, Pseudomonas aeruginosa $(n=1)$, Salmonella paratyphi A $(n=2)$, Salmonella paratyphi B $(n=1)$, Salmonella species $(n=3)$, Salmonella typhi $(n=5)$, Serratia marcescens $(n=1)$, Shigella boydii $(n=1)$, Shigella dysenteriae $(n=1)$, Shigella flexneri $(n=1)$, Shigella sonnei $(n=1)$ and Yersinia enterocolitica $(n=1)$ ] and 31 Gram-positive bacteria [Enterococcus avium $(n=1)$, Enterococcus casseliflavus $(n=2)$, Enterococcus duran $(n=1)$, Enterococcus faecalis $(n=17)$, Enterococcus gallinarum $(n=2)$, Enterococcus hirae $(n=1)$, Enterococcus mundtii $(n=1)$, Enterococcus raffinosus $(n=1)$, Staphylococcus aureus $(n=3)$ and Streptococcus group $\mathrm{G}(n=2)$ ].

Thermostabilization of PCR reagents. The specific primers that recognize all serogroups of $V$. cholerae were designed based on the lolB gene (GenBank accession no. AF227752.1), while the primers for detecting the virulence gene $c t x A$ were based on the sequence of the $c t x$ operon (GenBank accession no. X58785.1). IC primers were designed based on the SSP2 gene of Plasmodium falciparum (GenBank accession no. AL844509.2). The IC validates the PCR by verifying the absence of inhibiting factors which could lead to false-negative results. In this study, the IC was plasmid DNA containing sporozoite surface protein 2 (SSP2) from Plasmodium falciparum, an organism unrelated to $V$. cholerae. The forward and reverse primers were: lolB-F 5'-GTG CAT CTT GGT CGC GGT AG-3' and lolB-R 5'-GGC AAT CAC ACC AAG TCA CTC-3' (lolB gene); ctxA-F 5'-AAC TCA GAC GGG ATT TGT TAG GC- $3^{\prime}$ and $c t x A-R 5^{\prime}$-TCT CTG TAG CCC CTA TTA CGA TGT$3^{\prime}$ (ctxA gene); SSP2-F 5'-GGT ACC TTT ACC ACA AGT TAC AC-3' and SSP2-R 5'-TTC TCG ATC GTC TTC TGG AT-3' (IC). The primers were designed with Vector NTI Advance 9 software (Invitrogen).

Each PCR master reagent mix comprised $12 \mathrm{pmol}$ of each primer for the lolB gene, $16 \mathrm{pmol}$ of each primer for the $\operatorname{ctx} A$ gene, 4 pmol of each primer for the IC, $1 \times$ Green GoTaq Flexi Buffer (Promega), $1.5 \mathrm{mM} \mathrm{MgCl} \mathrm{Mg}_{2}$ (Promega), $0.16 \mathrm{mM}$ of each of the dNTPs (Fermentas), 1 U GoTaq DNA polymerase (Promega) and 40 pg IC DNA template. For the positive control, 660 pg extracted DNA of a $V$. cholerae reference strain was further added to the mixture, while the negative control received only PCR-grade (DNase-free) water (GIBCO) in place of the DNA. Trehalose as an enzyme stabilizer was added to the PCR reagent mix and the final mixture was subjected to a drying process for $2 \mathrm{~h}$ at $5.0^{-2} \mathrm{mBar}$ pressure using a Heto vacuum concentrator (Thermo Scientific Heto) connected to the LyoLab 3000 freeze-dryer (Thermo Scientific Heto). After that, the PCR tubes were stored in a sealed aluminium pouch.

Triplex PCR protocol and clinical evaluation. PCR was performed by addition of $18 \mu \mathrm{l} \mathrm{PCR}$-grade (DNase-free) water and $2 \mu \mathrm{l}$ DNA template to a tube containing thermostabilized PCR reagents. The mixture was vortexed briefly to dissolve the dried pellet. Each of the positive and negative control tubes received $20 \mu \mathrm{l}$ PCR-grade (DNasefree) water. The positive control was included to ensure the integrity of the PCR process, while the negative control was included to check for the presence of contaminating DNA. PCR amplification cycles were performed in a Bio-Rad DNA Engine thermal cycler with one initial denaturation step at $95{ }^{\circ} \mathrm{C}$ for $2 \mathrm{~min}, 35$ cycles of denaturation at $95{ }^{\circ} \mathrm{C}$ for $30 \mathrm{~s}$, annealing at $60{ }^{\circ} \mathrm{C}$ for $30 \mathrm{~s}$ and extension at $72{ }^{\circ} \mathrm{C}$ for $30 \mathrm{~s}$, followed by an extra annealing step at $60{ }^{\circ} \mathrm{C}$ for $30 \mathrm{~s}$ and a final extension step at $72{ }^{\circ} \mathrm{C}$ for $5 \mathrm{~min}$. The amplification products were resolved by $2.0 \%$ agarose gel electrophoresis, stained with ethidium bromide (Sigma), visualized under UV illumination and photographed using a ChemiImager 5500 image analyser (Alpha Innotech).

Stool obtained from a healthy volunteer was spiked with bacteria to mimic clinical stool specimens that would be obtained from 
diarrhoeal patients. Stool suspensions were prepared using a method described by McOrist et al. (2002) with modifications. Briefly, $10 \mathrm{~g}$ stool was added to $1 \times$ PBS buffer $(\mathrm{pH} 7.4)$ to make a $10 \%(\mathrm{w} / \mathrm{v})$ stool suspension. A volume of $200 \mu \mathrm{l}$ of the stool suspension was aliquoted into different $1.5 \mathrm{ml}$ tubes and spiked with a single colony of bacteria. The whole spiked stool suspension was inoculated into $5 \mathrm{ml}$ alkaline peptone water (APW) and incubated for $6 \mathrm{~h}$ at $37{ }^{\circ} \mathrm{C}$. Afterwards, $1 \mathrm{ml}$ of the APW culture was boiled for $5 \mathrm{~min}$ and pelleted by centrifugation at $5000 \mathrm{~g}$ for $10 \mathrm{~min}$. The resultant supernatant was used as template for the PCR assay.

The thermostabilized PCR was evaluated using DNA extracted from spiked stool specimens, composed of 102 positive strains of $V$. cholerae and 72 non- $V$. cholerae bacterial strains. The sensitivity, specificity, positive predictive value and negative predictive value were determined from the results based on a calculation previously reported (Spitalnic, 2004).

Determination of stability and analytical sensitivity of the thermostabilized PCR mix. The stability or shelf life of the thermostabilized triplex PCR assay was estimated using accelerated ageing techniques with elevated temperatures also known as the $\mathrm{Q}_{10}$ method. This technique is based on measuring storage of a medical device in elevated temperatures for a shorter period of time and mathematically correlating increased temperature with time (Clark, 1991). The thermostabilized PCR tubes were subjected to elevated temperatures of $37^{\circ} \mathrm{C}$ for up to 60 days and these tubes were tested periodically. The longest duration that the thermostabilized PCR maintained its activity was then calculated based on the formula provided by Clark (1991) to estimate its stability at ambient temperature $\left(24{ }^{\circ} \mathrm{C}\right)$.

The analytical sensitivity (LoD) of the thermostabilized PCR at the bacterial level was determined using $2 \mu$ template from lysates of serially diluted bacterial suspensions ranging from $10^{9}$ to $10^{4}$ c.f.u. $\mathrm{ml}^{-1}$. The final c.f.u. levels in the PCRs were from $2 \times 10^{6}$ c.f.u. to $2 \times 10^{1}$ c.f.u. The analytical sensitivity (LoD) at the genomic DNA level, on the other hand, was determined using various amounts of extracted genomic DNA ranging from $100 \mathrm{ng}$ to $100 \mathrm{fg}$ per PCR.

\section{RESULTS AND DISCUSSION}

The thermostabilized triplex PCR was standardized with known $V$. cholerae $\mathrm{O} 1$ and non-O1/non-O139 strains. The triplex PCR test is positive for a toxigenic strain of $V$. cholerae when all three amplicons of $300 \mathrm{bp}, 237 \mathrm{bp}$ and $150 \mathrm{bp}$ sizes are detected, which indicates the presence of the target $c t x A$ and lolB genes of $V$. cholerae and also the IC of PCR, respectively. Non-toxigenic $V$. cholerae is detected when only amplicons of $237 \mathrm{bp}$ (lolB gene) and $150 \mathrm{bp}$ (IC) are present, without the presence of a $300 \mathrm{bp}$ amplicon of the $\operatorname{ctxA}$ gene. The triplex PCR is considered a valid negative when only the PCR IC amplicon is observed (Fig. 1). If all three amplicons are absent, the PCR is defined as inhibited and the test should be repeated.

\section{Clinical evaluation of the thermostabilized triplex PCR}

The thermostabilized PCR assay was evaluated with 174 bacteria-spiked stool specimens and the diagnostic performance was determined. A representative agarose gel showing the results of the thermostabilized PCR using

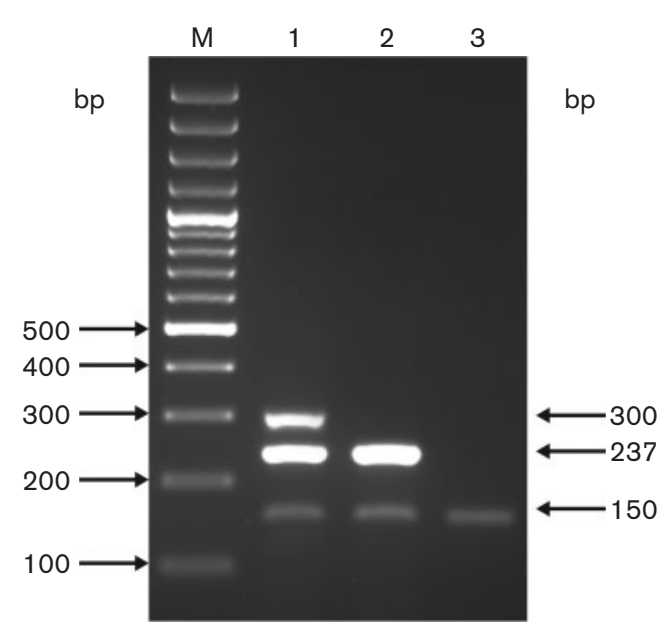

Fig. 1. Representative agarose gel electrophoresis of thermostabilized triplex PCR for cholera using known strains. Lanes: M, 100 bp DNA ladder (Fermentas); 1, V. cholerae O1; 2, V. cholerae non-O1/non-O139; 3, valid negative result.

spiked stool specimens is shown in Fig. 2. The thermostabilized PCR positively identified all toxigenic $V$. cholerae strains, where all the three genes (lolB, $\operatorname{ctx} A$ and the IC) were amplified, and all non-toxigenic $V$. cholerae strains, where only two genes (lolB and the IC) were amplified. All other Vibrio species or other bacteria tested negative, where only IC amplicons were detected. The overall analysis of the thermostabilized PCR showed $100 \%$ sensitivity, specificity, positive predictive value and negative predictive value. The high positive predictive value $(100 \%)$ suggested high test reliability and accuracy while the $100 \%$ negative predictive value showed perfect discrimination of non- $V$. cholerae strains.

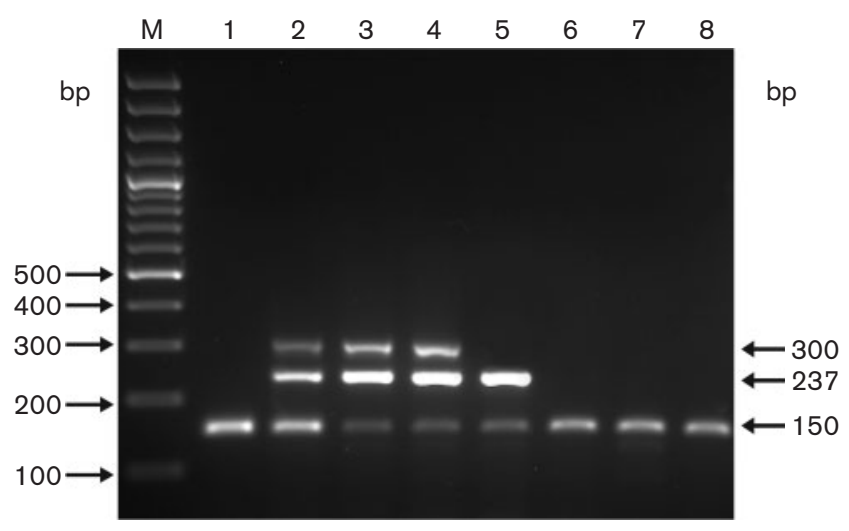

Fig. 2. Representative agarose gel electrophoresis of thermostabilized triplex PCR for cholera using bacteria-spiked stool specimens. Lanes: M, 100 bp DNA ladder; 1, PCR negative control; 2, PCR positive control; 3, V. cholerae O1; 4, V. cholerae O139; 5, V. cholerae non-O1/non-O139; 6, Vibrio species; 7, Escherichia coli; 8, Enterococcus faecalis. 


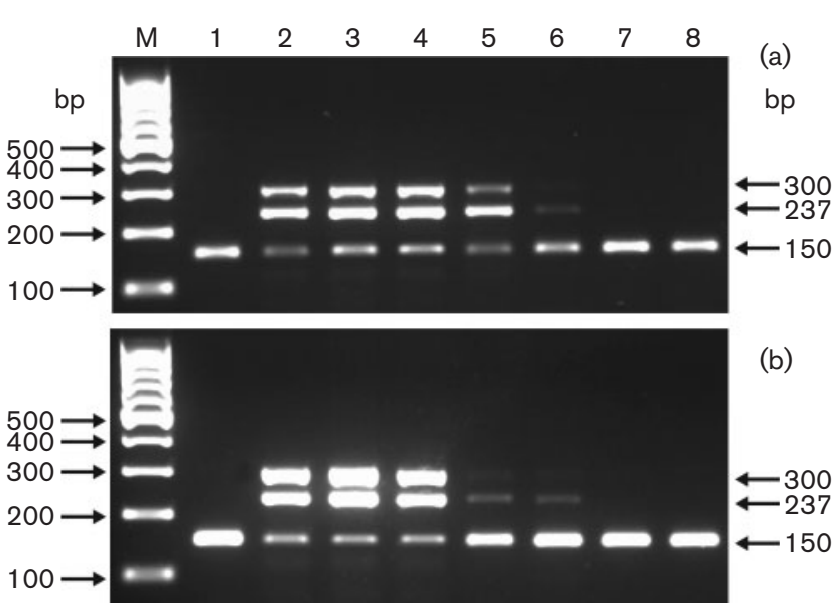

Fig. 3. Comparison of the analytical sensitivity of the thermostabilized triplex PCR mix (a) and conventional wet PCR (b). Various amounts of $V$. cholerae cells were used, ranging from $2 \times 10^{6}$ to $2 \times 10^{1}$ c.f.u. Lanes: M, 100 bp DNA ladder; 1, PCR negative control; 2, PCR positive control; $3,2 \times 10^{6}$ c.f.u.; $4,2 \times 10^{5}$ c.f.u.; $5,2 \times 10^{4}$ c.f.u.; $6,2 \times 10^{3}$ c.f.u.; $7,2 \times 10^{2}$ c.f.u.; $8,2 \times 10^{1}$ c.f.u.

\section{Determination of stability and analytical sensitivity}

The thermostabilized triplex PCR was evaluated for stability using the $\mathrm{Q}_{10}$ method and was found to be stable at an ambient temperature of $24{ }^{\circ} \mathrm{C}$ for 7.4 months when mathematically correlated with its stability at $37{ }^{\circ} \mathrm{C}$ (data not shown). Thus, this thermostabilized PCR mixture could be transported and stored without cold chain, an attribute useful for distributing this cholera test to many regions without the fear of degradation of reagents or reliability of the test. As a means to this end, trehalose was added as a stabilizer to protect and prevent conformation changes of the polymerase enzyme during dehydration. This strategy has also been similarly performed for mycobacteria and Salmonella typhi (Aziah et al., 2007; Klatser et al., 1998). However, the thermostabilization procedure was modified and optimized to suit the triplex
PCR in this study since the PCR parameters and compositions were different from those in previous studies. Trehalose protected and stabilized polymerase enzyme thus enables the cold chain-free nature of the thermostabilized PCR reagent mix. The thermostabilized PCR mix recorded a LoD of $2 \times 10^{4}$ c.f.u. at the bacterial cell level, and a LoD of $100 \mathrm{pg}$ DNA at the genomic DNA level (Figs 3 and 4), comparable to the conventional wet form. However, for the $c t x A$ gene, there was a one log lower sensitivity than for the lolB gene for both bacterial cell and DNA levels. The simplicity and ease-of-use of the thermostabilized PCR would outweigh its lesser sensitivity as compared to the more expensive real-time PCR. The LoD of the latter was reported at $100 \mathrm{fg}-1 \mathrm{pg}$ purified DNA and 14-20 c.f.u. $\mathrm{ml}^{-1}$ (Messelhäusser et al., 2010).

The thermostabilized PCR which specifically recognizes the lolB gene present in all serogroups of $V$. cholerae (Lalitha et al., 2008) has shown potential as a reliable test to detect all $V$. cholerae strains, even the sporadic cholera outbreaks by nonO1, non-O139 serogroups. Furthermore, the thermostabilized PCR developed here also detects the $\operatorname{ctxA}$ virulence gene. The $c t x A$ gene partly encodes cholera toxin, which is the main virulence factor responsible for the manifestation of diarrhoea and dehydration and is closely associated with clinical strains of $V$. cholerae (Lockman \& Kaper, 1983; Wachsmuth et al., 1994). Detection of cholera toxin has been previously described (Fields et al., 1992; Messelhäusser et al., 2010; Shirai et al., 1991) and has proved useful for identifying strains of $V$. cholerae often associated with epidemics. Thus the combined detection of the lolB and ctxA genes would enable efficient screening and detection of potential toxigenic strains of $V$. cholerae, which will prove useful especially during cholera outbreaks where rapid diagnosis is vital for outbreak management and containment.

Since cholera patient survivability depends on early treatment and patient management, faster diagnosis of cholera is important. Conventional methods such as culture and biochemistry tests for detecting $V$. cholerae are time-consuming while molecular diagnosis is becoming increasingly important for rapid detection. However, to

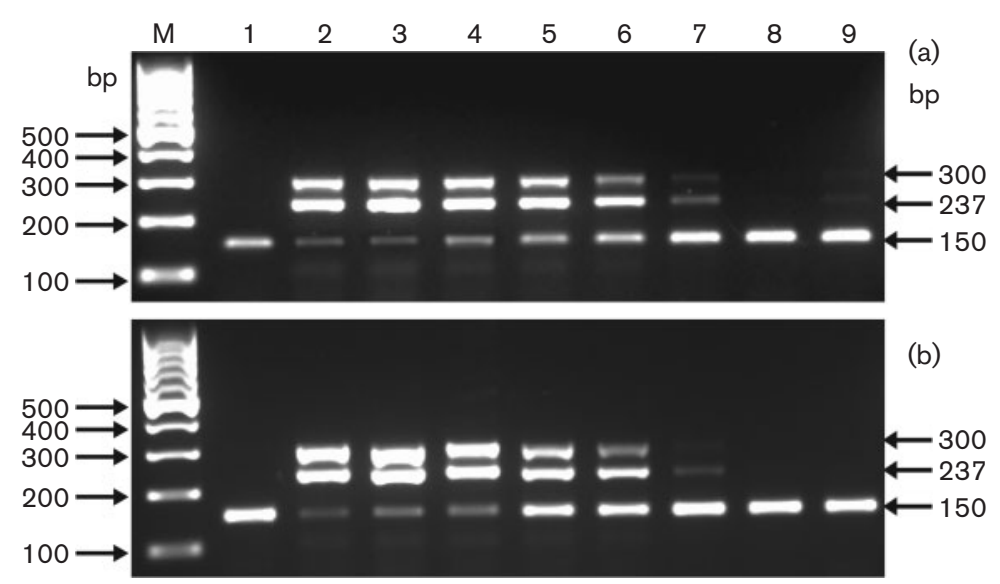

Fig. 4. Comparison of the analytical sensitivity of the thermostabilized triplex PCR mix (a) and conventional wet PCR (b). Various amounts of V. cholerae genomic DNA template were used, ranging from $100 \mathrm{ng}$ to $100 \mathrm{fg}$ DNA. Lanes: M, 100 bp DNA ladder; 1, PCR negative control; 2, PCR positive control; 3, 100 ng DNA; 4, 10 ng DNA; 5, 1 ng DNA; 6, 100 pg DNA; 7, 10 pg DNA; 8, 1 pg DNA; 9, 100 fg DNA. 
allow PCR tests to be more accessible to remote or resource-limited areas in which cholera outbreaks usually happen, this study has developed a cold chain-free, readyto-use PCR kit for detecting $V$. cholerae. Unlike traditional PCR, the thermostabilized PCR developed requires only two pipetting steps, which can help reduce cross-contamination generally caused by multiple pipetting steps. The thermostabilized PCR for cholera also incorporates an IC to detect inhibiting substances which could cause falsenegative results. Therefore, the thermostabilized PCR is suitable to be deployed in cholera outbreak sites for rapid detection and management of cholera outbreaks.

\section{ACKNOWLEDGEMENTS}

This study was supported by Research University grant (RU) of Universiti Sains Malaysia (USM), Malaysia. We would like to thank the following for providing the various bacterial strains used in this study: Center for Southeast Asia Studies, Kyoto University, Japan; Communicable Disease Hospital, Thandiarpettai, Chennai, India; National Public Health Laboratory, Ministry of Health, Malaysia; Institute for Medical Research (IMR), Malaysia; and the Medical Microbiology and Parasitology Laboratory, Universiti Sains Malaysia, Malaysia.

\section{REFERENCES}

Albert, M. J., Islam, D., Nahar, S., Qadri, F., Falklind, S. \& Weintraub, A. (1997). Rapid detection of Vibrio cholerae O139 Bengal from stool specimens by PCR. J Clin Microbiol 35, 1633-1635.

Aziah, I., Ravichandran, M. \& Ismail, A. (2007). Amplification of ST50 gene using dry-reagent-based polymerase chain reaction for the detection of Salmonella typhi. Diagn Microbiol Infect Dis 59, 373-377.

CDC (1999). Laboratory Methods for the Diagnosis of Epidemic Dysentery and Cholera. WHO/CDS/CSR/EDC/99.8. Atlanta, GA: Centers for Disease Control and Prevention.

Clark, G. S. (1991). Shelf Life of Medical Devices, April 1991. Food and Drug Administration Guidance Document.

Fields, P. I., Popovic, T., Wachsmuth, K. \& Olsvik, O. (1992). Use of polymerase chain reaction for detection of toxigenic Vibrio cholerae O1 strains from the Latin American cholera epidemic. J Clin Microbiol 30, 2118-2121.

Kaper, J. B., Morris, J. G., Jr \& Levine, M. M. (1995). Cholera. Clin Microbiol Rev 8, 48-86.

Klatser, P. R., Kuijper, S., van Ingen, C. W. \& Kolk, A. H. (1998). Stabilized, freeze-dried PCR mix for detection of mycobacteria. J Clin Microbiol 36, 1798-1800.

Koch, W. H., Payne, W. L., Wentz, B. A. \& Cebula, T. A. (1993). Rapid polymerase chain reaction method for detection of Vibrio cholerae in foods. Appl Environ Microbiol 59, 556-560.
Lalitha, P., Siti Suraiya, M. N., Lim, K. L., Lee, S. Y., Nur Haslindawaty, A. R., Chan, Y. Y., Ismail, A., Zainuddin, Z. F. \& Ravichandran, M. (2008). Analysis of lolB gene sequence and its use in the development of a PCR assay for the detection of Vibrio cholerae. J Microbiol Methods 75, 142-144.

Lipp, E. K., Rivera, I. N., Gil, A. I., Espeland, E. M., Choopun, N., Louis, V. R., Russek-Cohen, E., Huq, A. \& Colwell, R. R. (2003). Direct detection of Vibrio cholerae and ctxA in Peruvian coastal water and plankton by PCR. Appl Environ Microbiol 69, 36763680 .

Lockman, H. \& Kaper, J. B. (1983). Nucleotide sequence analysis of the A2 and B subunits of Vibrio cholerae enterotoxin. J Biol Chem 258, 13722-13726.

McOrist, A. L., Jackson, M. \& Bird, A. R. (2002). A comparison of five methods for extraction of bacterial DNA from human faecal samples. J Microbiol Methods 50, 131-139.

Messelhäusser, U., Colditz, J., Thärigen, D., Kleih, W., Höller, C. \& Busch, U. (2010). Detection and differentiation of Vibrio spp. in seafood and fish samples with cultural and molecular methods. Int $J$ Food Microbiol 142, 360-364.

Nandi, B., Nandy, R. K., Mukhopadhyay, S., Nair, G. B., Shimada, T. \& Ghose, A. C. (2000). Rapid method for species-specific identification of Vibrio cholerae using primers targeted to the gene of outer membrane protein OmpW. J Clin Microbiol 38, 4145-4151.

Rivera, I. G., Chowdhury, M. A., Huq, A., Jacobs, D., Martins, M. T. \& Colwell, R. R. (1995). Enterobacterial repetitive intergenic consensus sequences and the PCR to generate fingerprints of genomic DNAs from Vibrio cholerae O1, O139, and non-O1 strains. Appl Environ Microbiol 61, 2898-2904.

Shangkuan, Y. H., Show, Y. S. \& Wang, T. M. (1995). Multiplex polymerase chain reaction to detect toxigenic Vibrio cholerae and to biotype Vibrio cholerae O1. J Appl Bacteriol 79, 264-273.

Shirai, H., Nishibuchi, M., Ramamurthy, T., Bhattacharya, S. K., Pal, S. C. \& Takeda, Y. (1991). Polymerase chain reaction for detection of the cholera enterotoxin operon of Vibrio cholerae. J Clin Microbiol 29, 2517-2521.

Singh, D. V., Matte, M. H., Matte, G. R., Jiang, S., Sabeena, F., Shukla, B. N., Sanyal, S. C., Huq, A. \& Colwell, R. R. (2001). Molecular analysis of Vibrio cholerae O1, O139, non-O1, and non-O139 strains: clonal relationships between clinical and environmental isolates. Appl Environ Microbiol 67, 910-921.

Spitalnic, S. (2004). Test properties I: sensitivity, specificity, and predictive values. Hosp Physician 40, 27-31.

Wachsmuth, K., Blake, P. A. \& Olsvik, Ø. (1994). Vibrio cholerae and Cholera: Molecular to Global Perspectives. Washington, DC: American Society for Microbiology.

WHO (2009). Cholera: global surveillance summary, 2008. Wkly Epidemiol Rec 84, 309-324.

Yang, S. \& Rothman, R. E. (2004). PCR-based diagnostics for infectious diseases: uses, limitations, and future applications in acutecare settings. Lancet Infect Dis 4, 337-348. 